\title{
Nonverbal Expression of Psychological States in Psychiatric Patients
}

\author{
Heiner Ellgring \\ Max-Planck-Institut für Psychiatrie, Kraepelinstrasse 10, D-8000 München 40, Federal Republic of Germany
}

Summary. Nonverbal behavior, especially facial expression, appears as one of the most important means for communicating affective states. Studies on groups of psychiatric patients and control subjects are reported in which nonverbal behavior is analyzed from videotaped dialogues. Using a quantitative approach, results on facial behavior, speech, and gaze are described, which shed light on the expressive and communicative functions of nonverbal behavior. From longitudinal observations on depressed patients it emerged that individualspecific associations have to be taken into account for the relationship between expressive behavior and mood changes.

The predominance of facial behavior in the speaker role of an individual found in patients and control groups points to the integrated communicative function of the verbal and nonverbal elements. However, recovered schizophrenic patients exhibited a dissociation of these elements. Implications for our understanding of nonverbal communications are discussed.

Key words: Nonverbal communications - Facial expression Gaze behavior - Expression of mood states - Schizophrenia Depression - Psychopathology

\section{Introduction}

In what way are psychopathological states associated with specific changes in nonverbal behavior and is this behavior organized differently, thus indicating additional functional changes? In order to deal with these questions, observations on nonverbal behavior of schizophrenic patients and their relatives, of endogenous and neurotic depressed patients and of controls will be reported here.

At first, there seems to be an obvious relationship between internal mood states, emotions or affects, and our overt behavior. As socially living individuals, expressive behavior is one of the main sources of information when interpreting the actual motivational state of the other. As has been demonstrated in manifold ways since Darwin (1872) similar principles of expression hold for man and animals (Ploog 1980).

It may be argued that this means of communication loses its importance when replaced by language. However, numerous situations still exist where the nonverbal expression of affects and emotions are a central part of adult social interaction, not to mention infant-adult communication. For the understanding of an individual suffering from a psychiatric disorder, which nearly always includes emotional disturbances, expressive behavior seems to be relevant for various reasons.
As described by Ploog (1958, 1972), the breakdown of the social communication system in endogenous psychoses can lead to either blocking of motor expression, as is the case in catatonia, or to a disinhibition of drive states and their corresponding behavior. Thus, voluntary control mechanisms active in normal adult expressive behavior may lose their power during psychosis.

On the other hand, expressive behavior may also be regarded as a means whereby the patient can convey his actual states to the environment. Not only is depressive behavior as a "cry for help" (Arieti 1974) an expression of a mood state, but also a signal that will elicit behavior from the environment (Coyne 1976). In this way, nonverbal behavior functions as a means for communicating the altered affective state. This communicative function will, however, be effective only within a range where a person is not impaired too heavily by a psychosis. In order to communicate effectively, there must still be some choice for voluntarily activating parts of the behavioral repertoire. This may be the case for depression with the exception of extreme severity degrees - but less so in schizophrenic psychosis.

Expressive or nonverbal behavior is treated to some degree as a homogeneous set of signals or signs. Facial behavior, gaze, and gestures are all parts of a coherent repertoire. Together with speech and its accompanying vocal features it constitutes what appears for an observer to be a coordinated flow of complex communication. It must be remembered, however, that various behavioral elements are controlled by different neural mechanisms. They also represent different cognitive and emotional functions and are under different voluntary control. Gaze behavior, e.g., is closely connected to cognitive and attentional processes (Rutter 1981; Ellgring 1978) whereas facial behavior reflects affective processes as well as voluntary display according to socially determined rules (Ekman and Friesen 1969).

Four our understanding of the neural mechanisms underlying facial expression the behavior of patients suffering from Parkinson's disease deserves some consideration. Similar to depression, these patients exhibit little facial expression during normal conservation. Despite their inability to express feelings in their face, these patients are able to experience emotions and to move their facial muscles on request. Here, there is a dissociation of emotions and their expression. From the cerebral localisation of this disease, it can be concluded that expressive behavior is integrated not in the cortex but in the midbrain (Ploog 1980).

Being aware of the fact that it is always a set of signals and their continuous exchange between individuals that consti- 
tutes communication, we will focus our subsequent analysis on a few components of the behavioral repertoire. In order to compare individuals and situations, a quantitative approach has been chosen, i.e., an approach that tries to determine parameters for behavior coded by systematic observation. Behavioral measurements have been taken from video recordings ensuring observer reliability and registration of fast events like facial actions in slow motion.

Two aspects of nonverbal communication are dealt with: one addressing the question as to how depression as a homogeneous affective state correlates with changes in behavior of the nonverbal repertoire. The analysis focusses on the individual change of mood and behavior over time. The second aspect investigates the notion of a dissociation of coordinated behavior as a characteristic of psychoses. Various groups are compared with regard to the degree of coordination of nonverbal and verbal communicative behavior.

\section{Individual Specificity of Nonverbal Expression}

From intra-individual comparisons it appears that behavior correlates with the varying mood state during depression in an individual-specific way. In a longitudinal study 36 depressed patients (20 endogenous, ICD 296; 16 neurotic, ICD 300; 18 male, 18 female) and 9 controls ( 5 male, 4 female), with an age ranging from 24 to 64 years were repeatedly videotaped during interviews. For patients, an average period of 60 days (ranging from 25 to 152 days), for controls, periods ranging from 4 to 12 days were covered by observations. Subjective well-being of the patients was assessed by a visual analogue scale.

Various aspects of nonverbal behavior were analyzed for a selection of 120 out of a total of 506 interviews. Facial expression was measured by the Facial Action Coding System (FACS, Ekman and Friesen 1978) and specific parameters defined: facial repertoire, general facial activity and, as a specific activity, the occurrence of smiles. Speech related gestures were coded, and assessing on-off patterns only, gaze and speech activities were registered. Gaze and speech were analyzed for all of the interviews available. For neurotic depressed patients these consisted of 5 to 7 interviews, for endogenous patients more than 10 , up to 49 interviews. Details of the study have been reported elsewhere (Ellgring 1984).

In the present situation, two aspects of the results are relevant. Both indicate that the average relationship between subjective well-being and nonverbal behavior may obscure very strong associations on the individual level.

For about $70 \%$ of the patients, only 1 or 2 of the total of 6 nonverbal parameters assessed substantially changed along with improvement of the mood state (see Fig. 1).

As can be seen, less than $10 \%$ of the patients showed changes in 4,5 , or 6 of these parameters. Only those changes that exceeded variations of the behavior found in repeated observations of normal controls were considered. On the other hand, only 3 of 36 depressed patients $(=8 \%$ ) showed no substantial change in any of the behavioral parameters.

Generally, the frequency of smile $(61 \%)$ and the amount of gaze at the other $(58 \%)$ showed an increase with improvement. For general facial activity $(31 \%)$ and speech activity $(33 \%)$, this was the case in about $1 / 3$ of the cases, whereas the facvial repertoire $(22 \%)$ and speech related gestures $(14 \%)$ rarely changed according to our criteria. There was no clear-

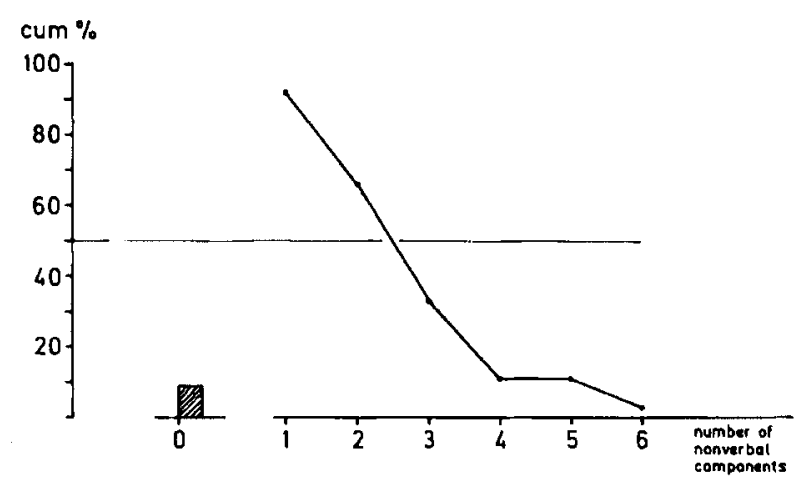

Fig.1. Substantial changes of nonverbal behavioral components. Percentage of depressed patients (cum $\%, n=36$ ), exhibiting substantial change in 0 resp. 1 or more components of nonverbal behavior with improvement of subjective well-being
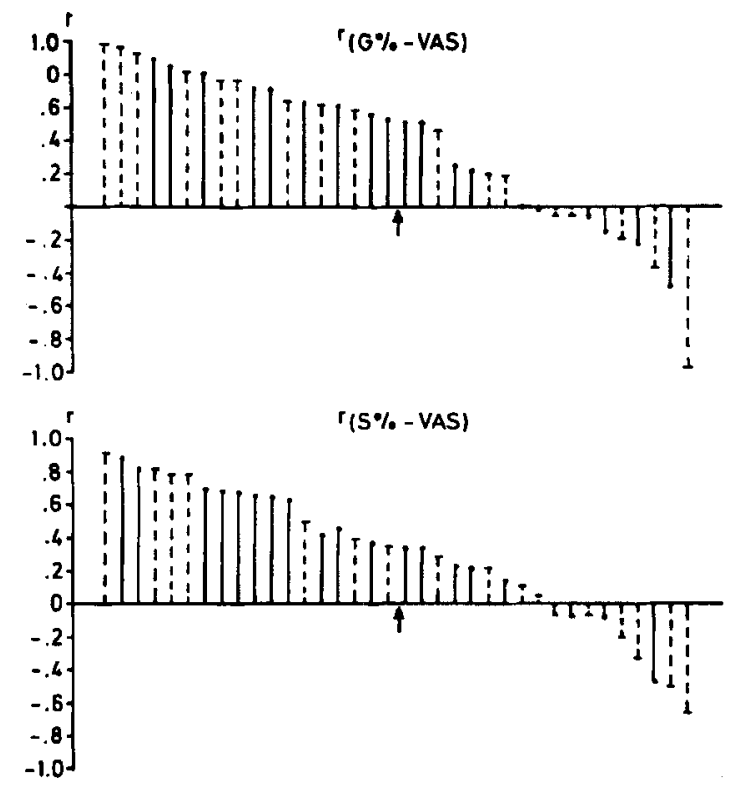

Fig. 2. Individual correlations between subjective well-being (Visual Analogue Scale, VAS) and relative amount of gaze (G \%) resp. speech activity (S\%). $\longrightarrow$ Endogenous (>10 interviews), - - neurotic depressed patients (6-7 interviews)

cut hierarchy amongst these aspects of behavior. From this one has to assume that they are connected to the depressed mood in a way represented by a logical "or" relation. Gaze or facial expression or other behavior do change in an individualspecific way along with an improvement in subjective wellbeing. The individual correlations of speech and gaze behavior with subjective well-being strengthen this argument. As can be seen from Fig. 2, there was a broad range of individual correlations, most of them located on the positive side.

The average correlation between subjective well-being and the relative amount of gaze was $r=0.58$; the corresponding correlation for the relative amount of speech activity $r=0.38$. However, these average correlations obscure the finding of very strong individual relationships especially for gaze behavior. Both behavioral aspects correlated independently with subjective well-being $(r=0.07)$. 
The general results correspond to those reported in the literature (Jones and Pansa 1979; Rutter 1984). When using a group statistic approach only minor changes of nonverbal behavior are found in psychiatric patients. This contradiction to clinical impression dissolves when analyzing at the individual level. Comparable to physiological reactions (Lacey 1967), there is individual specificity of expressive behavior in depression. This individual-specific "usage" of nonverbal elements as an expression of a depressed state indicates some peculiarities of nonverbal communication.

The fact that various parts of the nonverbal repertoire change during depression in different individuals seem to contradict the notion of "universality" in nonverbal behavior (see, e.g., Eibl-Eibesfeldt 1984; Ploog 1980). However, from a communication point of view this specificity could be seen as the basis for a "differential" understanding. Persons acquainted with the patient recognize behavioral changes when comparing the known individual in various situations. The relevant information can then be filtered out, disregarding other elements.

\section{Behavioral Coordination}

One of the most fascinating features of nonverbal behavior is the organisation of its elements as a highly coordinated behavioral flow, smoothly exchanged between interactants (von Cranach 1971). An obvious example is the coordination of gaze with speech. A speaker tends to turn the gaze away when starting to speak or when confronted with a task, and he returns the gaze on completion of the speech or answer (Kendon 1967; Ellgring 1984). For facial behavior, a different coordination is of interest; as facial expression is activated at a high level when one communicates verbally to another person. It is, however, reduced to a very low level during emotional or nonemotional imagery, and during observation of emotional films (Ellgring et al. 1985; Vanger and Ellgring 1985). It is therefore probable that facial expression is closely connected to verbal or vocal communication.

During psychosis, a dissolution of otherwise coordinated behavior has been observed by Ploog (1972). The question being investigated empirically, then, is if a dissolution can be found for the verbal-facial coordination reported above.

Again facial behavior was coded from the videotaped interactions. In addition, it was noted whether facial action took place during an utterance (speaker role) or during the utterance of the other person (listener role). From the previous experimental results, a higher proportion of facial expression should occur during those parts of the dialogue where the person takes the speaker role than when taking the listener role.

For each of 10 endogenous, 10 neurotic patients and 9 controls, three interviews were examined from the sample mentioned before. In addition, for a group of 10 schizophrenic patients (ICD 295; 4 male, 6 female, age ranging from 17 to 46 years, median 24 years), facial behavior was analyzed when interacting with their relatives at the end of their clinical stay. Information on relapse within 1 year was available. Relatives' facial behavior was assessed twice: once when interacting with the patient discussing a point of disagreement and once when being interviewed by a clinician about the patient. The interview took place shortly after admission of the patient and was used to rate positive and negative attitudes of the relatives towards the patient (Hahlweg et al. in preparation).

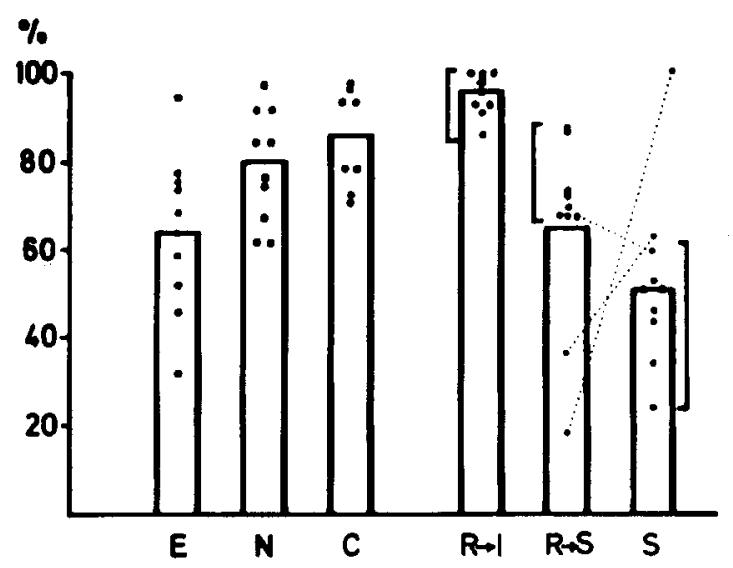

Fig.3. Proportion of facial expression when taking the speaker role. Averages and individual data (dotted). $\mathrm{E}=$ endogenous, $\mathrm{N}=$ neurotic, $\mathrm{C}=$ controls, $\mathrm{R}-\mathrm{I}=$ relatives in interview, $\mathrm{R}-\mathrm{S}=$ relatives with recovered schizophrenics, $S=$ recovered schizophrenics. Lines connect dyads of patients and relatives (see text)

Table 1. Statistical comparisons between groups with regard to their average proportions of facial activity in speaker/listener role. $P$-values from $U$-tests. $\mathbf{E}=$ endogenous, $\mathbf{N}=$ neurotic depressed patients, $\mathrm{C}=$ controls, $\mathbf{R}-\mathbf{I}=$ relatives in interview, $\mathrm{R}-\mathrm{S}=$ relatives interacting with recovered schizophrenic patients, $S=$ recovered schizophrenic patients

\begin{tabular}{|c|c|c|c|c|c|c|}
\hline & $\mathrm{E}$ & $\mathbf{N}$ & $\mathrm{C}$ & R-I & R-S & $\mathbf{S}$ \\
\hline $\mathrm{E}$ & - & 0.07 & $<0.01 * *$ & $<0.001^{* * *}$ & 0.91 & $<0.11$ \\
\hline $\mathbf{N}$ & & - & 0.24 & $<0.01^{* *}$ & 0.17 & $<0.001^{* * *}$ \\
\hline C & & & - & 0.05 & $<0.01^{* *}$ & $<0.001^{* * *}$ \\
\hline R-I & & & & - & $<0.001^{* * *}$ & $<0.001^{* * *}$ \\
\hline R-S & & & & & - & 0.06 \\
\hline $\mathbf{S}$ & & & & & & - \\
\hline
\end{tabular}

The relative amount of facial expression when in the speaker or the listener role was the critical variable. If facial expression is independent of the verbal communication, a proportion of $50 \%$ would be expected. Figure 3 contains average and single values for the proportion of facial expression being displayed in the speaker role. Values higher than $50 \%$ indicated the expected association of facial expression with verbal communication. Values lower than $50 \%$ indicated a dissolution of both communication modes.

As can be seen from Fig. 3, there were, on average, substantial differences between groups with regard to facial-verbal association. Controls and relatives in the interview displayed more than $70 \%$ of their facial activity in the speaker role. For neurotic depressed patients, this average proportion was slightly lower. Endogenous depressed patients showed a similar proportion as relatives interacting with recovered schizophrenic patients. The clearly lowest average proportion (about 50\%) was displayed by schizophrenic patients in this interaction.

Table 1 gives the levels of significance for single comparisons between groups using $U$-tests after a highly significant Kruskal Wallis analysis $(H=33.5, d f=5, P<0.01)$.

Apart from the group comparisons, the single values, marked in Fig. 3, were examined in more detail. Despite the averages being quite similar for endogenous patients and relatives interacting with schizophrenic patients, there were sub- 
stantial differences in the kind of variability. Although for endogenous patients there was a broad range of proportion values, only two of the relatives showed exceptional data, the other covering a narrow range. The corresponding values of the patients, on the other hand, were extremes in the opposite direction. Obviously, these cases were complementary but not the remainder. Interestingly enough, in two cases of the three in the sample a high rating of negative attitudes predicted relapse that did not however occur. The third of these cases is indicated by the dotted line in Fig. 3. Again values were located at the extremes of the respective groups though not as dramatically as for the other two. (For further analyses of facial expression, see Ellgring et al. 1985).

So there is the general tendency for coordination of facial expression and verbal communication in normals and also in most of the depressed patients. The only exception was the group of recovered schizophrenics. Here, a random association of facial and verbal communication emerged. In addition, the exceptional data of two dyads indicate compensatory processes between patients and relatives which need further clarification.

\section{Discussion}

From the analysis of facial expression we found evidence for a tendency of the nonverbal behavior to dissociate from verbal communication in psychoses. There was a slight tendency for this dissolution in endogenous depressed patients and an even stronger one for recently recovered schizophrenic patients. For normal controls and relatives of patients, there was, in contrast, a very close association of facial expression and verbal communication. One can only speculate on the exceptional data from the group of schizophrenic patients interacting with their relatives. One interpretation would be that a high proportion of facial expression of the relative when listening to the patient (corresponding to a low proportion in the speaker role) may serve as some emotional feedback given to the patient. This could be a protective factor for those interactions where a high degree of rated negative attitude would otherwise predict a relapse. Other explanations are, however, possible and need further empirical investigation.

From the coordination of facial expression to verbal behavior it is clear that under normal conditions both serve communicative functions in an interrelated way (Ploog 1980). Facial expression complements the verbal content at an emotional level, however they seem to be dissociated in the recovered schizophrenic. It could be that this kind of dissociation may contribute to the perception of disturbed communication in this group.

The individual specificity of behavioral changes found in depressed patients points to an inconsistency with regard to the universality of expressive behavior. In the expression of children and in the perception of emotional expression, crosscultural universality has been reported (Eibl-Eibesfeldt 1984). For the patients' behavior, however, this universality seems only to be partly true, with an individual-specific association of behavior and mood changes emerging. It is not known whether this is also the case for other adults in varying emotional states.

When considering an economical way for transmitting emotional information, this individual-specific "usage" of nonverbal behavior has several advantages. Only part of the repertoire has to change during a depressed state. Moreover, since the specific effectors become apparent only for those who are able to observe the individual and compare behavior over the time course, acquaintances have an easier access to this information. From this it follows that the level of transmitting emotional or affective information varies depending on the relationship between interactants. The same behavior, thus, has a different meaning for persons knowing the individual than for strangers. In this way, the same behavior informs on different levels of acuity depending on the closeness of the relationship. Individual-specific expression of mood, therefore, seems to be an effective way of communcation.

\section{References}

Arieti S (1974) Affective disorders: Manic-depressive psychosis and psychotic depression. In: Arieti S, Brody EB (eds) American Handbook of Psychiatry, vol 3. Adult clinical psychiatry. Basic Books, New York, pp 449-490

Coyne JC (1976) Toward an interactional description of depression. Psychiatry $39: 28-40$

Cranach M von (1971) Die nichtverbale Kommunikation im Kontext des kommunikativen Verhaltens. In: Jahrbuch der Max-PlanckGesellschaft zur Förderung der Wissenschaften. Max-PlanckGesellschaft, Göttingen, pp 104-148

Darwin C (1965) The expression of the emotions in man and animals. (1872) The University of Chicago Press, Chicago

Eibl-Eibesfeldt I (1984) Die Biologie des menschlichen Verhaltens. Grundriß der Humanethologie. Piper, München

Ekman P, Friesen WV (1969) The repertoire of nonverbal behavior Categories, origins, usage, and coding. Semiotica 1:49-98

Ekman P, Friesen WV (1978) Facial Action Coding System. Consulting Psychologists Press, Palo Alto, CA, USA

Ellgring H (1984) Nonverbale Kommunikation im Verlauf der Depression - Zum Ausdruck der Stimmung und des Befindens in Mimik, Blickzuwendung, Sprechen und Gestik. (Habilitationsschrift). Justus-Liebig-Universität, Giessen

Ellgring H, Hahlweg K, Feinstein E, Dose M (1985a) Affective behaviour of relatives during CFT and family interaction. Paper presented at the Ringberg Symposium "The Impact of Family Interaction Research on our Understanding of Psychopathology". Schloß Ringberg

Eligring H, Nagel U, Ferstl R (1985b) Facial expression during imagery and communication. Paper presented at the International Conference on the Meaning of Faces. Cardiff, 1985

Jones JH, Pansa M (1979) Some nonverbal aspects of depression and schizophrenia occurring during the interview. J Nerv Ment Dis $167: 402-409$

Kendon A (1967) Some functions of gaze direction in social interaction. Acta Psychol 26:22-63

Lacey JI (1967) Somatic response patterning and stress: Some revisions of activation theory. In: Appley MH, Trumbull R (eds) Psychological Stress. Appleton-Century-Crofts, New York, pp $14-42$

Ploog D (1958) Úber das Hervortreten angeborener Verhaltensweisen in akuten schizophrenen Psychosen. Psychiatr Neurol 136: $157-164$

Ploog D (1972) Breakdown of the social communication system: A key process in the development of schizophrenia? Prospects for Research on schizophrenia, neurosciences. Research Prog Bull $10: 394-395$

Ploog D (1980) Der Ausdruck der Gemütsbewegungen bei Mensch und Tieren. In: Max-Planck-Gesellschaft (ed) Jahrbuch 1980. Vanderhöck and Ruprecht, Göttingen, pp 66-97

Rutter DR (1984) Looking und seeing: The role of visual communication in social interaction., Wiley, New York

Vanger $\mathrm{Ph}$, Ellgring $\mathrm{H}$ (1985) Facial activity and intensity of emotional experience. Paper presented at the International Conference on the Meaning of Faces, Cardiff, Wales, June 1985

Received December 10, 1985 